\title{
Stereotactic radiosurgery for central neurocytomas: an international multicenter retrospective cohort study
}

\author{
Yi-Chieh Hung, MD, ${ }^{1-3}$ Cheng-Chia Lee, MD, PhD, ${ }^{4,5}$ Huai-che Yang, MD, ${ }^{4,5}$ \\ Nasser Mohammed, MD, MCh, ${ }^{1}$ Kathryn N. Kearns, BS, ${ }^{1}$ Shi-Bin Sun, MD, ${ }^{6}$ David Mathieu, MD, \\ Charles J. Touchette, MD, ${ }^{7}$ Ahmet F. Atik, MD, ${ }^{8}$ Inga S. Grills, MD, ${ }^{9}$ Bryan Squires, MD, ${ }^{9}$ \\ Dale Ding, MD, ${ }^{10}$ Brian J. Williams, MD, ${ }^{10}$ Mehran B. Yusuf, MD, ${ }^{11}$ Shiao Y. Woo, MD, ${ }^{11}$ \\ Roman Liscak, MD,12 Jaromir Hanuska, MD, ${ }^{13}$ Jay C. Shiao, MD, MPH, MSc, ${ }^{14}$ \\ Douglas Kondziolka, MD, ${ }^{15}$ L. Dade Lunsford, MD, ${ }^{16}$ Zhiyuan Xu, MD, ${ }^{1}$ and \\ Jason P. Sheehan, MD, PhD'
}

1Department of Neurological Surgery, University of Virginia Health System, Charlottesville, Virginia; ${ }^{2}$ Department of Neurosurgery, Department of Surgery, Chi-Mei Medical Center, Tainan, Taiwan; ${ }^{3}$ Department of Recreation and Healthcare Management, Chia Nan University of Pharmacy and Science, Tainan, Taiwan; ${ }^{4}$ Department of Neurosurgery, Neurological Institute, Taipei Veterans General Hospital, Taipei, Taiwan; ${ }^{5}$ School of Medicine, National Yang-Ming University, Taipei, Taiwan; ${ }^{6}$ Gamma Knife Center, Beijing Neurosurgical Institute, Beijing Tiantan Hospital, Capital University of Medical Sciences, Beijing, China; ${ }^{\prime}$ Université de Sherbrooke, Centre de Recherche du Centre Hospitalier Universitaire de Sherbrooke, Québec, Canada; ${ }^{8}$ Department of Neurosurgery, Cleveland Clinic Foundation, Cleveland, Ohio; ${ }^{9}$ Department of Radiation Oncology, William Beaumont Hospital, Royal Oak, Michigan; Departments of ${ }^{10}$ Neurosurgery and ${ }^{11}$ Radiation Oncology, University of Louisville, Kentucky; Departments of ${ }^{12}$ Stereotactic and Radiation Neurosurgery and ${ }^{13}$ Neurosurgery, Na Homolce Hospital, Prague, Czech Republic; ${ }^{14}$ Department of Radiation Oncology, University of Colorado Cancer Center, Aurora, Colorado; ${ }^{15}$ Department of Neurosurgery, NYU Langone Health System, New York, New York; and ${ }^{16}$ Department of Neurosurgery, University of Pittsburgh, Pittsburgh, Pennsylvania

OBJECTIVE Central neurocytomas (CNs) are uncommon intraventricular tumors, and their rarity renders the risk-tobenefit profile of stereotactic radiosurgery (SRS) unknown. The aim of this multicenter, retrospective cohort study was to evaluate the outcomes of SRS for CNs and identify predictive factors.

METHODS The authors retrospectively analyzed a cohort of patients with CNs treated with SRS at 10 centers between 1994 and 2018. Tumor recurrences were classified as local or distant. Adverse radiation effects (AREs) and the need for a CSF shunt were also evaluated.

RESULTS The study cohort comprised 60 patients (median age 30 years), 92\% of whom had undergone prior resection or biopsy and $8 \%$ received their diagnosis based on imaging alone. The median tumor volume and margin dose were $5.9 \mathrm{~cm}^{3}$ and $13 \mathrm{~Gy}$, respectively. After a median clinical follow-up of 61 months, post-SRS tumor recurrence occurred in 8 patients (13\%). The 5 - and 10-year local tumor control rates were $93 \%$ and $87 \%$, respectively. The 5 - and 10 -year progression-free survival rates were $89 \%$ and $80 \%$, respectively. AREs were observed in 4 patients $(7 \%)$, but only 1 was symptomatic (2\%). Two patients underwent post-SRS tumor resection (3\%). Prior radiotherapy was a predictor of distant tumor recurrence $(p=0.044)$. Larger tumor volume was associated with pre-SRS shunt surgery $(p=0.022)$.

CONCLUSIONS Treatment of appropriately selected CNs with SRS achieves good tumor control rates with a reasonable complication profile. Distant tumor recurrence and dissemination were observed in a small proportion of patients, which underscores the importance of close post-SRS surveillance of $\mathrm{CN}$ patients. Patients with larger $\mathrm{CNs}$ are more likely to require shunt surgery before SRS.

https://thejns.org/doi/abs/10.3171/2020.1.JNS191515

KEYWORDS central neurocytoma; Gamma Knife radiosurgery; stereotactic radiosurgery; intracranial neoplasms

ABBREVIATIONS ARE = adverse radiation effect; $\mathrm{CN}=$ central neurocytoma; EBRT = external-beam radiation therapy; $\mathrm{GTR}=$ gross-total resection; IRRF = International Radiosurgery Research Foundation; PFS = progression-free survival; RANO = Response Assessment in Neuro-Oncology; RTOG = Radiation Therapy Oncology Group; SRS = stereotactic radiosurgery; STR = subtotal resection.

SUBMITTED May 30, 2019. ACCEPTED January 27, 2020.

INCLUDE WHEN CITING Published online April 3, 2020; DOI: 10.3171/2020.1.JNS191515. 
$\mathrm{C}$ ENTRAL neurocytomas (CNs) are rare central nervous system tumors, representing only $0.1 \%-0.5 \%$ of intracranial neoplasms. ${ }^{1-3}$ According to the WHO classification, CNs are categorized as grade II tumors. ${ }^{4-7}$ CNs are usually observed near the foramen of Monro in the lateral ventricles. Due to their common intraventricular location, the initial clinical presentation of these lesions is often headaches and obstructive hydrocephalus. CNs primarily occur in young adults, with $70 \%$ of cases diagnosed between the 2 nd and 4 th decades of life. ${ }^{3}$ Treatment strategies for CNs include resection, with or without radiation therapy, or biopsy followed by radiation therapy. The 5-year local tumor control rate is approximately $70 \%-100 \% .^{8-10}$ Upfront stereotactic radiosurgery (SRS) achieves good tumor control rates for biopsy-proven CNs, even in some cases of large tumors. ${ }^{11}$ The tumor control rates are high with both conventional fractionated external-beam radiation therapy (EBRT) and SRS, but the incidence of adverse radiation effects (AREs) may be lower after SRS (3\%-20\% with SRS vs $60 \%$ with EBRT). ${ }^{412-14}$ However, while SRS ameliorates the risk of AREs with its focused targeting and steep dose falloff, the potential for distant (out-field) recurrence remains with this therapeutic modality.

Tumor recurrence after treatment of CNs is associated with neurological morbidity and mortality. ${ }^{12,14,15}$ Because of the rarity of CNs and the limited statistical power of predominantly single-center SRS studies to date, the incidence and risk factors of distant recurrence are not well defined in the literature. Additionally, the risk factors for CSF diversion procedures are not well characterized in CN patients treated with SRS. Therefore, the aims of this multicenter, retrospective cohort study were to 1) determine the rates of local tumor control and progression-free survival (PFS) after SRS for CNs, 2) characterize AREs associated with CNs treated with SRS, and 3) identify risk factors for CSF shunt surgery in SRS-treated CN patients.

\section{Methods \\ Patient Population}

Patients who underwent SRS for CNs between 1994 and 2018 at 10 institutions participating in the International Radiosurgery Research Foundation (IRRF) were included in the study. The contribution from each site was as follows: Beaumont Health System $(n=2)$, Beijing Tiantan Hospital $(n=8)$, Cleveland Clinic $(n=4)$, Na Homolce General Hospital $(n=2)$, Taipei Veterans General Hospital $(n=32)$, University of Colorado $(\mathrm{n}=1)$, University of Louisville $(\mathrm{n}=$ 2), University of Sherbrooke $(n=4)$, University of Virginia $(n=7)$, and New York University $(n=1)$. Data were collected retrospectively under the institutional review boardapproved protocols of each respective institution. As this was a retrospective study, patient consent was not required.

A template database with selected variables was created and sent to all participating centers. De-identified data from each contributing institution were screened for inconsistencies, verified for compliance with current standards of patient privacy and personal information protection, and pooled by an independent third party. The pooled data were transmitted to the first and senior authors for analysis on behalf of the IRRF. Any uncertainties or ambiguities in the data were addressed by the contributing center.

\section{Baseline Data and Variables}

The baseline data comprised patient demographics, treatments before SRS, tumor characteristics, and SRS parameters. The patient demographics included age at SRS, sex, and presenting symptoms. Treatments before SRS included the need for CSF shunt surgery, extent of tumor resection (gross-total resection [GTR], subtotal resection [STR], or biopsy), and time interval from tumor surgery to SRS. Tumor characteristics included location and volume. SRS variables included the number of tumors treated, treatment volume, margin dose, maximum dose, and isodose line.

\section{Composition of the Study Cohort}

The inclusion criteria for this study were patients with 1) CNs that were treated with single-session SRS, 2) sufficient baseline data to assess demographic information, and 3) $\geq$ 3 months of radiological and clinical follow-up. After excluding 2 patients who were treated with hypofractionated CyberKnife radiosurgery and 1 with no clinical follow-up, the study cohort comprised 60 eligible patients.

Table 1 details the baseline patient and tumor characteristics of the study cohort. Twenty-nine patients (48\%) were male. The median age at the time of SRS was 30 years (range 5-71 years). The most common clinical symptom was headache $(n=45 / 60,75 \%)$. The most common tumor location was the lateral ventricle $(n=58 / 60,97 \%)$. The median time from the last tumor surgery to SRS was 6 months (range 1-120 months). Five patients underwent pre-SRS EBRT.

\section{SRS Technique}

SRS was performed using either the Gamma Knife (Elekta AB) or the CyberKnife (Accuray Inc.); the specific models used differed by year and availability at each institution. The general protocol for Gamma Knife SRS has been previously described. ${ }^{16,17}$ Briefly, with the patient under local or monitored anesthesia, a Leksell model $\mathrm{G}$ stereotactic frame (Elekta AB) was affixed to the calvaria. The CyberKnife SRS procedure was frameless and performed without anesthesia. Thin-slice T1- and T2weighted MRI sequences including gadolinium contrastenhanced images were obtained for treatment planning.

Treatment parameters and dose plans were dictated, in part, by tumor location and size, distance between the tumor and normal brain, and previous EBRT. Exposure of the bilateral fornices to radiation was minimized when feasible. Dose planning and SRS delivery were performed by a multidisciplinary team of radiation oncologists, medical physicists, and neurosurgeons.

SRS was performed after resection in 50 patients; there were 7 cases of tumor recurrence after GTR (3 tumors were treated with upfront SRS to the resection bed, and 4 tumors were treated due to tumor recurrence) and 43 cases of adjuvant therapy after STR and as an upfront treatment in 10 patients (4 tumors were treated with upfront SRS for residual tumors and the remaining 39 tumors were treated 
TABLE 1. Patient characteristics: descriptive statistics (total cases $=60$ )

\begin{tabular}{|c|c|}
\hline Variable & Value \\
\hline Male sex & $29(48 \%)$ \\
\hline Median age at SRS, yrs (range) & $30(5-71)$ \\
\hline \multicolumn{2}{|l|}{ Clinical symptoms } \\
\hline Asymptomatic & $8(13 \%)$ \\
\hline Headache & $45(75 \%)$ \\
\hline Nausea/vomiting & $22(37 \%)$ \\
\hline Memory impairment & $12(20 \%)$ \\
\hline Visual disturbance & $7(12 \%)$ \\
\hline Seizure & $11(18 \%)$ \\
\hline Ataxia & $9(15 \%)$ \\
\hline \multicolumn{2}{|l|}{ Location of lesions } \\
\hline Lateral ventricle & $52(87 \%)$ \\
\hline Lateral \& 3rd ventricles & $3(5 \%)$ \\
\hline Lateral \& 4th ventricles & $2(3 \%)$ \\
\hline Lateral, 3rd, \& 4th ventricles & $1(2 \%)$ \\
\hline Only 3rd ventricle & $2(3 \%)$ \\
\hline Previous shunt & $22(37 \%)$ \\
\hline Tumor $<6 \mathrm{~cm}^{3}$ & $7 / 31(23 \%)$ \\
\hline Tumor $>6 \mathrm{~cm}^{3}$ & $15 / 29(52 \%)$ \\
\hline \multicolumn{2}{|l|}{ Diagnosis } \\
\hline Imaging (no histology) & $5(8 \%)$ \\
\hline Previous op & $55(92 \%)$ \\
\hline \multicolumn{2}{|l|}{ Previous op type $(n=55)$} \\
\hline GTR & $7 / 55(13 \%)$ \\
\hline STR & $43 / 55(78 \%)$ \\
\hline Biopsy & $5 / 55(9 \%)$ \\
\hline Median time from op to SRS, mos (range) & $6(1-120)$ \\
\hline Previous EBRT & $5(8 \%)$ \\
\hline \multicolumn{2}{|l|}{ Reason for SRS } \\
\hline Regrowth after total removal & $7(12 \%)$ \\
\hline Adjuvant therapy after partial removal & $43(72 \%)$ \\
\hline Upfront treatment w/ or w/o biopsy & $10(17 \%)$ \\
\hline \multicolumn{2}{|l|}{ SRS parameter } \\
\hline \multicolumn{2}{|l|}{ No. of treated tumors } \\
\hline 1 & $53(88 \%)$ \\
\hline 2 & $4(7 \%)$ \\
\hline 3 & $3(5 \%)$ \\
\hline Median treatment vol, $\mathrm{cm}^{3}$ (range) & $5.9(0.2-48.9)$ \\
\hline Median margin dose, Gy (range) & $13(10-30)$ \\
\hline Median maximum dose, Gy (range) & $23(15-66)$ \\
\hline Median isodose line, \% (range) & $56(30-67)$ \\
\hline Median clinical follow-up, mos (range) & $61(3-241)$ \\
\hline Median imaging follow-up, mos (range) & $53(3-241)$ \\
\hline
\end{tabular}

Values presented as number (\%) unless otherwise indicated.

because of tumor recurrence). Five cases were biopsy-proven CNs, and the other 5 cases of CNs were diagnosed by neuroimaging alone without histopathological confirmation. The number of tumors treated with SRS was 1,2 , and 3 in $53(88 \%), 4(7 \%)$, and $3(5 \%)$ patients, respectively. Patients who had 2 or 3 SRS targets were those with multiple remnants after surgery or new tumors after surgery. For patients with multiple SRS-treated lesions, only the param- eters of the largest tumor were selected for analysis. The median treatment volume, margin dose, and maximum dose were $5.9 \mathrm{~cm}^{3}$ (range $\left.0.2-48.9 \mathrm{~cm}^{3}\right), 13$ Gy (range 10 $30 \mathrm{~Gy}$ ), and $23 \mathrm{~Gy}$ (range 15-66 Gy), respectively (Table 1).

\section{Clinical and Neuroimaging Follow-Up}

Clinical and neuroimaging assessments were generally performed at 6-month intervals for the first 2 years after SRS and then yearly thereafter. When MRI was contraindicated, CT with contrast was performed instead. Additional neuroimaging was performed in patients with neurological changes during the follow-up period. All images were reviewed by the treating clinical team. Whenever possible, clinical follow-up was obtained concurrently with routine neuroimaging follow-up. When in-person follow-up was not feasible, clinical and neuroimaging data from other institutions or physicians were transmitted to the treating institution for review. All follow-up clinical and neuroimaging data were compared with data obtained at the time of SRS.

\section{Outcomes}

The radiological tumor outcomes after SRS were evaluated using the criteria proposed by the Response Assessment in Neuro-Oncology (RANO) group. ${ }^{18}$ Tumor control was defined by the summation of stable disease, partial response, and complete response. Recurrence was categorized as local versus distant recurrence. Local recurrence was defined as progressive enlargement of the target lesions. Distant recurrence was defined as progression of an existing nontarget lesion or de novo formation of a new lesion. Local tumor control was defined as survival without local recurrence. PFS was defined as survival without local or distant recurrence. AREs and death were recorded. The Radiation Therapy Oncology Group (RTOG) Late Radiation Morbidity Scoring Schema ${ }^{19}$ was used to evaluate the neurotoxicity of SRS.

\section{Statistical Analysis}

All statistical analyses were performed using IBM SPSS (version 24.0, IBM Corp.). Descriptive statistics for continuous and categorical variables are reported as median or mean and frequency or percentage, respectively. Univariate and multivariate analyses were performed using the Cox proportional hazards regression model to determine risk factors for overall tumor control, survival without local recurrence, and survival without distant recurrence. Hazard ratios and 95\% confidence intervals were calculated for each variable.

The Kaplan-Meier method was used to compute PFS, local tumor control, and survival without distant recurrence over time. Comparisons of the aforementioned survival outcomes between patients with versus without pre-SRS EBRT were performed using the log-rank test. The binary logistic regression method was used to identify factors associated with pre-SRS shunt surgery. Odds ratios and $95 \%$ confidence intervals were calculated for each variable. Baseline variables in univariate analysis with a $\mathrm{p}$ value $<0.15$ were entered into a multivariate binary logistic regression model to identify independent predictors 
TABLE 2. Outcomes after SRS (in 60 patients)

\begin{tabular}{lc}
\hline \multicolumn{1}{c}{ Variable } & No. of Patients (\%) \\
\hline Clinical status & \\
Improved & $9(15 \%)$ \\
No change & $49(82 \%)$ \\
\hline Deteriorated/new neurological deficit & $3(5 \%)$ \\
\hline Image outcome: RANO criteria* & \\
Complete response & $4(7 \%)$ \\
Partial response & $42(70 \%)$ \\
Stable disease & $10(17 \%)$ \\
Progressive disease (local recurrence) & $4(7 \%)$ \\
\hline Distant recurrence & $4(7 \%)$ \\
\hline Overall recurrence & $8(13 \%)$ \\
\hline ARE & $4(7 \%)$ \\
\hline Grade I: mild/asymptomatic $(n=4)$ & $4(100 \%)$ \\
\hline Shunt after SRS & $2(3 \%)$ \\
\hline Death & $1(2 \%)$ \\
\hline
\end{tabular}

* Complete response, disappearance of all CNS target lesions sustained for at least 4 weeks; partial response, at least a $30 \%$ decrease in the sum longest diameter of CNS target lesions, using as reference the baseline sum longest diameter sustained for 4 weeks; stable disease, neither sufficient shrinkage to qualify for partial response nor sufficient increase to qualify for progressive disease, taking as reference the smallest sum longest diameter while on study; and progressive disease, at least a $20 \%$ increase in the sum longest diameter of CNS target lesions, taking as reference the smallest sum on the study. ${ }^{18}$

of pre-SRS shunt surgery. Statistical significance was defined as $\mathrm{p}<0.05$, and all tests were two-tailed.

\section{Results}

\section{Radiological and Clinical Outcomes}

Table 2 summarizes the radiological and clinical out- comes of the study cohort, which comprised $60 \mathrm{CN}$ patients treated with SRS. Clinically, 58 patients (97\%) showed stability or improvement of their pre-SRS symptoms. With respect to the targeted lesions, the rates of complete response, partial response, stable disease, and progressive disease (i.e., local recurrence) were $7 \%,(\mathrm{n}=$ $4 / 60), 70 \%(n=42 / 60), 17 \%(n=10 / 60)$, and $7 \%(n=4 / 60)$, respectively. Distant recurrence was observed in an additional 4 patients $(7 \%)$. In total, tumor recurrence (local or distant) occurred in 8 patients (13\%).

The median radiological and clinical follow-up durations after SRS were 53 and 61 months, respectively. The actuarial rates of PFS (i.e., survival without local or distant recurrence) at 2, 5, 8, and 10 years after were $96 \%$, $89 \%, 85 \%$, and $80 \%$, respectively (Fig. 1A). The actuarial rates of survival without local recurrence (i.e., local tumor control) at 2, 5, 8, and 10 years were $98 \%, 93 \%, 93 \%$, and $87 \%$, respectively (Fig. 1B). The actuarial rates of survival without distant recurrence at 2, 5, 8, and 10 years were $98 \%, 96 \%, 92 \%$, and $82 \%$, respectively (Fig. 1C).

\section{Post-SRS Treatment of Recurrent Tumors}

Additional treatment after SRS was performed in 5 patients $(8 \%)$ with local or distant recurrence (Table 3). Of the patients with local recurrence $(n=4)$, tumor resection was performed in 2, both of whom had tumor control at follow-up durations of 28 and 43 months. The remaining 2 patients with local recurrence opted for observation, and their tumors were radiologically stable at the last followup (durations of 15 and 38 months). Of the patients with distant recurrence $(n=4)$, repeat SRS was performed in 3, and 1 patient opted for conservative management. Tumor control was achieved at last follow-up in 3 patients with distant recurrences, including 2 treated with repeat SRS and 1 conservatively managed, at follow-up durations of 24,28 , and 156 months; the tumor in remaining patient
(A) Progression free survival (overall recurrence, $n=8 / 60$ )

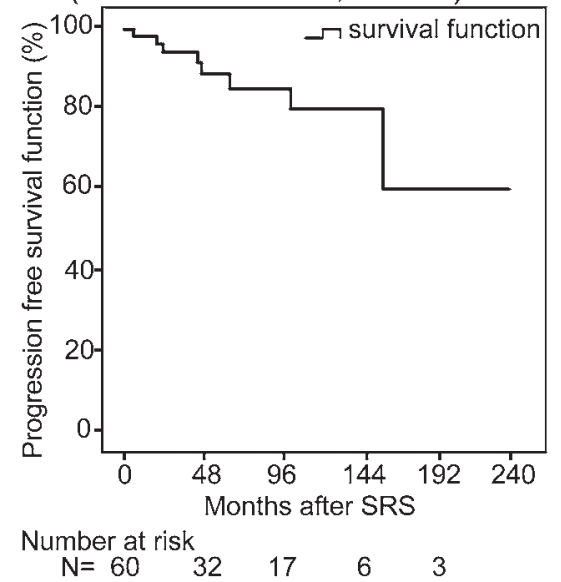

(B) Survival without local recurrence

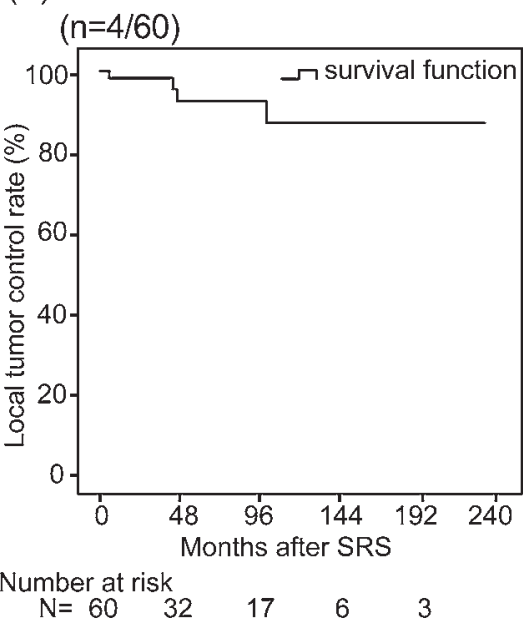

(C) Survival without distant recurrence

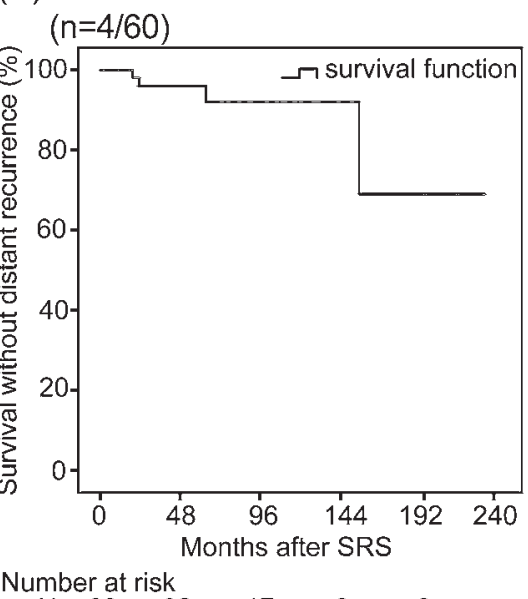

FIG. 1. A: Kaplan-Meier analysis of PFS after SRS. The actuarial rates for this endpoint at 2, 5, 8, and 10 years were $96 \%, 89 \%$, $85 \%$, and $80 \%$, respectively. B: Kaplan-Meier analysis of survival without local recurrence (local tumor control) after SRS. The actuarial rates for this endpoint at 2, 5, 8, and 10 years were $98 \%, 93 \%, 93 \%$, and $87 \%$, respectively. C: Kaplan-Meier analysis of survival without distant recurrence after SRS. The actuarial rates for this endpoint at 2, 5, 8, and 10 years were $98 \%, 96 \%, 92 \%$, and $82 \%$, respectively. 
TABLE 3. Treatment of recurrent cases

\begin{tabular}{|c|c|c|c|c|c|c|}
\hline Case No. & Time to Recurrence (mos) & 2nd Treatment & Treatment Vol $\left(\mathrm{cm}^{3}\right)$ & Margin Dose (Gy) & Final Tumor Control & PFS (mos) \\
\hline \multicolumn{7}{|c|}{ Local recurrence } \\
\hline 23 & 46 & Surgery & & & Yes & 28 \\
\hline 34 & 104 & Surgery & & & Yes & 43 \\
\hline 46 & 48 & No & & & Yes & 38 \\
\hline 52 & 6 & No & & & Yes & 15 \\
\hline \multicolumn{7}{|c|}{ Distant recurrence } \\
\hline 2 & 66 & Repeat SRS & 7.3 & 18 & Yes & 156 \\
\hline 12 & 20 & Repeat SRS & $\begin{array}{l}2.1 \\
0.8 \\
0.4 \\
0.03\end{array}$ & $\begin{array}{l}12 \\
20 \\
23 \\
18\end{array}$ & $\mathrm{No}^{*}$ & \\
\hline 33 & 162 & No & & & Yes & 28 \\
\hline 56 & 24 & Repeat SRS & 0.8 & 15 & Yes & 24 \\
\hline
\end{tabular}

* Patient died of disseminated disease 21 months after SRS.

who underwent repeat SRS progressed, and the patient died of disseminated disease at 21 months of follow-up.

\section{Risk Factors for Distant Tumor Recurrence}

Table 4 details the univariate and multivariate Cox proportional hazards regression models for distant tumor recurrence. Only pre-SRS EBRT was significantly associated with distant recurrence in the univariate analysis $(\mathrm{p}$ $=0.038$ ). Pre-SRS EBRT remained the only independent predictor of distant recurrence in the multivariate analysis (HR 20.43, 95\% CI 1.89-383.00; $\mathrm{p}=0.044$ ). The actuarial rates of survival without distant recurrence at 1,2 , and 5 years were $100 \%, 67 \%$, and $67 \%$, respectively, with preSRS EBRT versus $100 \%, 100 \%$, and $98 \%$, respectively, without pre-SRS EBRT. Patients without pre-SRS EBRT had significantly higher rates of survival without distant recurrence ( $p=0.004$; Fig. 2).

\section{Risk Factors for Pre-SRS Shunt Surgery in CN Patients}

Table 5 details the univariate and multivariate logistic regression for pre-SRS shunt surgery, which was performed in 22 patients (37\%). Only SRS treatment volume was significantly associated with shunt surgery in the univariate analysis $(\mathrm{p}=0.012)$. Treatment volume remained

TABLE 4. Risk factors for distant tumor recurrence

\begin{tabular}{|c|c|c|c|c|c|c|c|c|}
\hline \multirow[b]{2}{*}{ Factor } & \multicolumn{2}{|c|}{ Distant Tumor Recurrence } & \multicolumn{3}{|c|}{ Univariate Analysis } & \multicolumn{3}{|c|}{ Multivariate Analysis* } \\
\hline & No $(n=56)$ & Yes $(n=4)$ & $\mathrm{HR}$ & $95 \% \mathrm{Cl}$ & p Value & $\mathrm{HR}$ & $95 \% \mathrm{Cl}$ & $p$ Value \\
\hline Male & $28(50 \%)$ & $1(25 \%)$ & 0.49 & $0.05-4.85$ & 0.544 & & & \\
\hline Mean age at SRS, yrs (SD) & $32(14)$ & $34(15)$ & 1.03 & $0.96-1.11$ & 0.415 & & & \\
\hline Pre-SRS shunt & $19(34 \%)$ & $3(75 \%)$ & 4.47 & $0.46-43.35$ & 0.197 & & & \\
\hline Pre-SRS tumor op & $51(91 \%)$ & $4(100 \%)$ & 23.60 & & 0.715 & & & \\
\hline Total tumor removal & $7(12 \%)$ & $0(0 \%)$ & 0.04 & & 0.736 & & & \\
\hline Tumor biopsy & $4(7 \%)$ & $1(25 \%)$ & 3.65 & $0.33-40.49$ & 0.292 & & & \\
\hline Pre-SRS EBRT & $3(5 \%)$ & $1(25 \%)$ & 19.18 & $1.19-310.21$ & 0.038 & 20.43 & $1.89-383.00$ & 0.044 \\
\hline Mean time surgery to SRS, mos (SD) & $17(27)$ & $15(14)$ & 1.00 & $0.97-1.04$ & 0.956 & & & \\
\hline Multiple targets at SRS $†$ & $7(12 \%)$ & $0(0 \%)$ & 0.04 & $0.00-21,821.00$ & 0.627 & & & \\
\hline Mean treated vol, $\mathrm{cm}^{3}(\mathrm{SD})$ & $9(9)$ & $16(22)$ & 1.01 & $0.94-1.09$ & 0.814 & & & \\
\hline Mean margin dose, Gy (SD) & $14(3)$ & $15(2)$ & 1.14 & $0.92-1.42$ & 0.223 & & & \\
\hline Mean maximum dose, Gy (SD) & $25(7)$ & $28(5)$ & 1.04 & $0.96-1.13$ & 0.336 & & & \\
\hline Mean isodose line, \% (SD) & $54(7)$ & $55(6)$ & 0.97 & $0.85-1.11$ & 0.634 & & & \\
\hline Mean image follow-up, mos (SD) & $72(57)$ & $78(54)$ & 0.95 & $0.91-1.00$ & 0.056 & 0.95 & $0.89-1.00$ & 0.064 \\
\hline
\end{tabular}

Values presented as number (\%) unless otherwise indicated. Boldface type indicates statistical significance $(p<0.05)$. Cox regression, $n=4 / 60$.

* Only factors with $p<0.15$ in the univariate analysis were listed in the multivariate analysis.

$\dagger$ More than 1 tumor treated during SRS. 


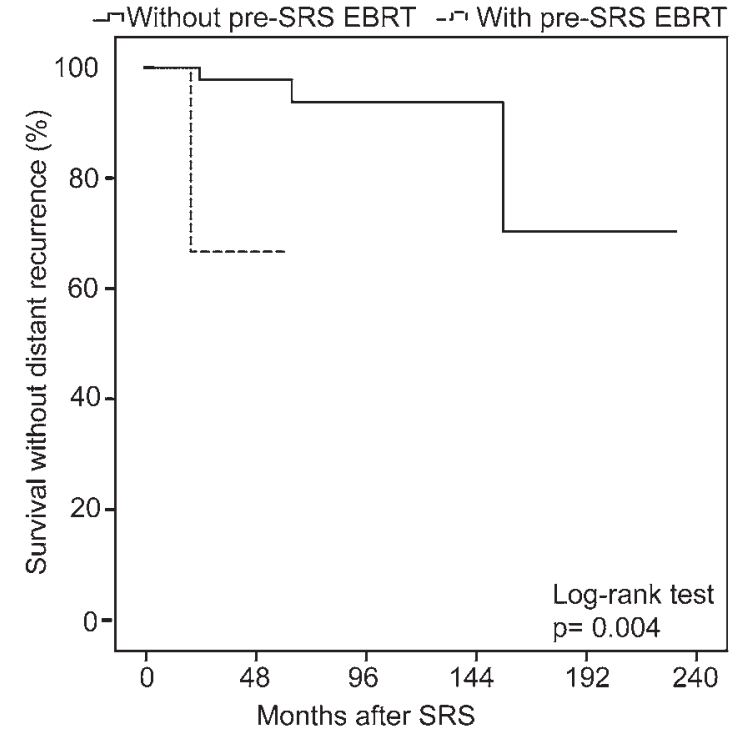

Number at risk

\begin{tabular}{|c|c|c|}
\hline $\begin{array}{l}\text { Without pre-SRS EBRT } \\
\qquad \mathrm{N}=55\end{array}$ & 17 & 6 \\
\hline pre- & & \\
\hline
\end{tabular}

FIG. 2. Kaplan-Meier analysis of survival without distant recurrence comparing CN patients with $(100 \%, 67 \%, 67 \%$ at 1,2 , and 5 years, respectively) versus without $(100 \%, 100 \%, 98 \%$ at 1,2 , and 5 years, respectively) pre-SRS EBRT. The rates of distant recurrence were significantly higher in CNs treated with pre-SRS EBRT ( $p=0.004$, log-rank test).

the only independent risk factor for pre-SRS shunt surgery in the multivariate analysis (OR 1.08, 95\% CI 1.01-1.15; $\mathrm{p}=0.022$ ). Two patients with pre-SRS CSF shunts underwent shunt revision surgery after SRS $(n=2 / 22,9 \%)$.

\section{Complications}

AREs occurred in 4 patients (7\%), which radiologically manifested as new T2-weighted hyperintensities in the peritumoral brain region. An ARE was only symptomatic in 1 patient (2\%), who developed transient diplopia. Overall, 3 patients had worsening symptoms, including the aforementioned patient with a symptomatic ARE. Another patient developed progressive headaches due to multiple distant recurrences. Despite repeat SRS for the recurrent tumors, the patient died at 21 months of progressive disease. The third patient experienced monocular visual deterioration due to retinal vein occlusion without evidence of ARE or tumor recurrence.

\section{Discussion}

Surgery and radiation therapy are the typically employed treatments to manage CNs, whereas chemotherapy is reserved for rare cases of recurrent, progressive, or disseminated disease..$^{15,20,21}$ Resection of CNs is the first-line treatment, which affords 5-year local tumor control rates of $70 \%-100 \%{ }^{8,10,22}$ However, GTR can be associated with high complication rates due to the surrounding critical neurovascular structures., ${ }^{4,8,9,23-25}$ In addition, many CNs are noted to be hypervascular on angiography, ${ }^{26}$ which could result in considerable intraoperative or postoperative hemorrhage..$^{14,17,27}$

The supplementation of stereotactic radiotherapy with adjuvant EBRT affords tumor control rates that are comparable with GTR ${ }^{8}{ }^{89}$ Compared with radiotherapy, SRS reduces the radiation dose to the surrounding normal brain tissue and critical structures. ${ }^{13,28}$ In this multicenter, retrospective analysis, we present the largest cohort of CNs treated by SRS to date to evaluate the rates and risk factors for local tumor control, distant tumor recurrence, and preSRS CSF shunt surgery. ${ }^{43-25}$

\section{Local Tumor Control}

Rades and Fehlauer performed a meta-analysis that included patients with CNs treated by surgery with or without EBRT to compare different strategies for achieving tumor control. ${ }^{8}$ In this report, STR alone had significantly lower 5-year rates of local tumor control (46\%) compared with other single or combination treatment approaches. Supplementing STR with adjuvant EBRT increases the 5 -year local tumor control rate (83\%), and these outcomes are comparable to GTR with or without adjuvant EBRT. ${ }^{9}$ Even for atypical neurocytomas, the additional adjuvant EBRT could still increase the 5-year local tumor control rate from $5 \%-7 \%$ to $65 \%-70 \% .{ }^{6.9}$

CNs are more frequently diagnosed in young adults. Thus, the benefits of a more focused, rather than widefield, radiotherapy seem appealing. ${ }^{10}$ Chen et al. evaluated the long-term outcomes of postoperative EBRT in a cohort of 67 CNs. ${ }^{12}$ Thirty-eight patients experienced late neurotoxicity $(60 \%)$, including 10 with grade 2 or 3 toxicities, as defined by the Common Terminology Criteria for Adverse Events. The most common ARE in that series was shortterm memory impairment.

Given the focal and well-demarcated nature of most CNs, SRS has become an important treatment option for these lesions. ${ }^{28}$ In the present study, the SRS afforded high rates of local tumor control $(93 \%$ at 5 years, $87 \%$ at 10 years) and PFS (89\% at 5 years, $80 \%$ at 10 years). SRS also exhibited an excellent safety profile in our cohort. Four patients developed AREs (7\%), but only 1 patient was symptomatic (mild diplopia). In other published studies of SRS for residual or recurrent $\mathrm{CNs}$, the 5-year local tumor control rate ranged from $90 \%$ to $94 \%$, with neurotoxicity in $3 \%-20 \%$ of patients (Table 6 ). ${ }^{14,29-31}$

The local tumor control rates of EBRT and SRS were compared in a previous case series (5-year local control rates of $87 \%$ and $100 \%$, respectively) (13 $^{13}$ and a review (overall local control rate of $88 \%$ and $93 \%$, respectively). ${ }^{28} \mathrm{Un}-$ like SRS for other intracranial neoplasms, the margin dose did not correlate with local tumor control in this study (Table 6). ${ }^{14,30-32}$

\section{Distant Tumor Recurrence and Disseminated Disease}

Survival without distant tumor recurrence was observed in $96 \%$ and $82 \%$ at 5 and 10 years, respectively. This endpoint has not been rigorously assessed in the current literature. ${ }^{14,17,22,30}$ One study proposed the use of postoperative EBRT for CNs to prevent distant recurrence. ${ }^{8}$ Our Cox pro- 
TABLE 5. Predictors for pre-SRS shunt surgery

\begin{tabular}{|c|c|c|c|c|c|c|c|c|}
\hline \multirow[b]{2}{*}{ Factor } & \multicolumn{2}{|c|}{ Any Shunt Surgery } & \multicolumn{3}{|c|}{ Univariate Analysis } & \multicolumn{3}{|c|}{ Multivariate Analysis* } \\
\hline & No $(n=38)$ & Yes $(n=22)$ & OR & $95 \% \mathrm{Cl}$ & p Value & OR & $95 \% \mathrm{Cl}$ & $p$ Value \\
\hline Male & $18(47 \%)$ & $11(5 \%)$ & 1.11 & $0.39-3.18$ & 0.844 & & & \\
\hline Mean age at treated SRS, yrs (SD) & $30(14)$ & $28(15)$ & 1.02 & $0.98-1.06$ & 0.260 & & & \\
\hline Headache & $26(68 \%)$ & $19(86 \%)$ & 2.92 & $0.72-11.81$ & 0.132 & 2.18 & $0.50-9.02$ & 0.310 \\
\hline Pre-SRS tumor surgery & $35(92 \%)$ & $20(91 \%)$ & 0.86 & $0.13-5.57$ & 0.872 & & & \\
\hline Total tumor removal & $3(8 \%)$ & $4(18 \%)$ & 2.67 & $0.53-13.38$ & 0.233 & & & \\
\hline Tumor biopsy & $2(5 \%)$ & $3(14 \%)$ & 2.91 & $0.44-19.13$ & 0.266 & & & \\
\hline Pre-SRS EBRT & $4(11 \%)$ & $1(5 \%)$ & 0.41 & $0.04-3.87$ & 0.432 & & & \\
\hline Mean time surgery to SRS, yrs (SD) & $15(28)$ & $21(24)$ & 1.01 & $0.99-1.03$ & 0.437 & & & \\
\hline Multiple targets at SRS† & $4(11 \%)$ & $3(14 \%)$ & 1.34 & $0.27-6.64$ & 0.718 & & & \\
\hline Mean treated volume, $\mathrm{cm}^{3}(\mathrm{SD})$ & $7(6)$ & $15(13)$ & 1.09 & $1.02-1.16$ & 0.012 & 1.08 & $1.01-1.15$ & 0.022 \\
\hline Mean margin dose, Gy (SD) & $14(3)$ & $14(2)$ & 0.92 & $0.74-1.15$ & 0.483 & & & \\
\hline Mean maximum dose, Gy (SD) & $35(5)$ & $27(10)$ & 1.03 & $0.96-1.12$ & 0.397 & & & \\
\hline Mean isodose line, \% (SD) & $55(6)$ & $53(7)$ & 0.96 & $0.89-1.04$ & 0.370 & & & \\
\hline Mean image follow-up, mos (SD) & $70(52)$ & $48(64)$ & 1.00 & $0.99-1.01$ & 0.634 & & & \\
\hline
\end{tabular}

portional hazards regression showed that pre-SRS EBRT was the only predictor of distant recurrence. We hypothesize that CNs previously treated with EBRT may represent a subset of tumors that are more biologically aggressive and/or radioresistant. However, the number of cases of distant recurrence was limited, so our analysis for predictors of this endpoint should be interpreted with caution.

SRS might carry a higher risk for distant recurrence than EBRT due to its relatively smaller treatment field. However, the supposition that EBRT is more effective at preventing $\mathrm{CN}$ dissemination is not endorsed by the available literature. ${ }^{28}$ Our findings support rigorous, long-term follow-up after SRS for CNs to facilitate early radiological identification of distant recurrence.

\section{Shunt Surgeries in Patients With CNs}

The need for CSF diversion is particularly relevant to the management of intraventricular tumors such as CNs. In addition to relieving the symptoms of obstructive hydrocephalus associated with CNs, CSF shunting could also aid in the management of post-SRS complications, such as ventriculomegaly, intraventricular hemorrhage, and peritumoral edema. ${ }^{17,30,33,34}$ However, one must also consider the clinical and neurological implications of shunt-related complications, such as malfunction and infection. ${ }^{4,17,30}$

In the present study, $37 \%$ of $\mathrm{CN}$ patients underwent preSRS CSF shunt surgery. Treatment volume was the only risk factor for pre-SRS shunt surgery. This finding is consistent with the greater likelihood of larger intraventricular tumors to impair or obstruct normal CSF flow. Pre-SRS shunt surgery was more frequently performed in patients with a tumor volume $\geq 6 \mathrm{~cm}^{3}$ at the time of SRS $(52 \%$ vs $32 \%, \mathrm{p}=0.019$ ). Two of the 22 patients subsequently un- derwent post-SRS shunt revisions (9\%). The tumors in the 2 patients with post-SRS CSF shunt revision were 9 and $12 \mathrm{~cm}^{3}$ in volume at SRS, and they both showed partial responses to SRS based on RANO criteria (> 30\% reduction in tumor diameter). For large CNs, shunt surgery may not be needed after a radical tumor surgery. Nevertheless, this result should be applied according to the uniqueness of each patient.

\section{Limitations}

Several limitations of this study should be recognized. Five patients were diagnosed with CNs based on clinical and radiographic evidence alone, without obtaining histopathology. It is possible that the tumors in these patients were pathologies other than CNs. Due to the retrospective design of this study, our findings and conclusions are subject to the inherent selection, treatment, and referral biases of each contributing institution and its physicians, although pooling data across multiple centers may somewhat mitigate the severity of these biases.

Some of the patients included in the present cohort have been previously evaluated in single-center studies from participating IRRF centers. ${ }^{17,31}$ However, for the purposes of this study, all patient data were updated as a new data set, which provides sufficient case numbers for statistical analysis. The inclusion of more patients in this multicenter study provides increased statistical power for determining risk factors, compared with prior single-center studies with smaller sample sizes. Since all of the patients in our cohort were treated with SRS, we are unable to compare the SRS outcomes for CNs to those of alternate management options, such as resection (initial or repeat), EBRT (upfront or postoperative), and surveillance. 


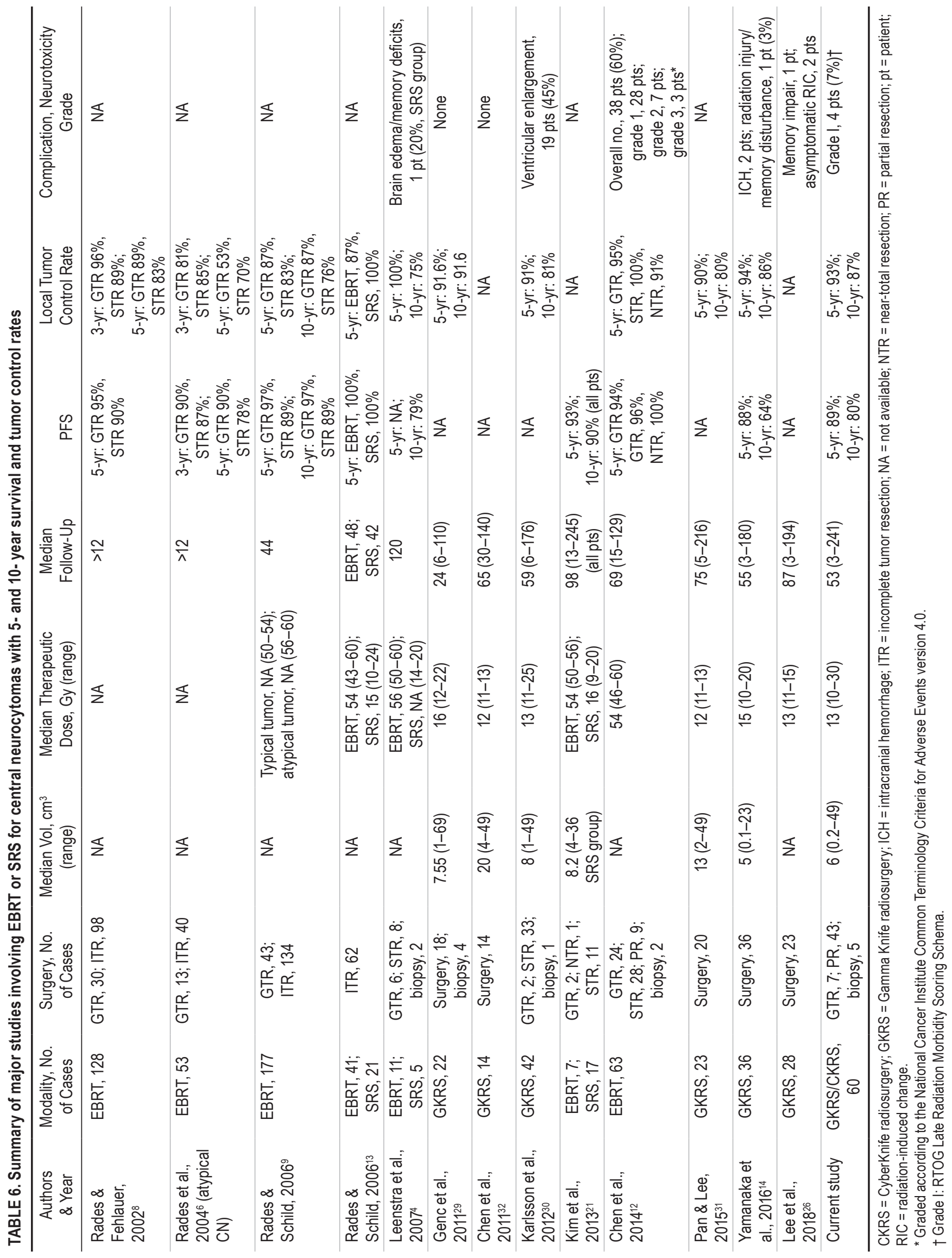




\section{Conclusions}

SRS affords a favorable risk-to-benefit profile for the management of CNs, with high rates of local tumor control and low risks of symptomatic complications. A modest proportion of $\mathrm{CN}$ patients will have distant tumor recurrence after SRS, so long-term surveillance after treatment is prudent. Distant recurrence occurs more frequently in patients with previously irradiated CNs, which suggests that the tumors in these patients are more biologically aggressive or radioresistant. Patients with large CNs are more likely to require CSF shunt placement before SRS.

\section{References}

1. Hassoun J, Söylemezoglu F, Gambarelli D, et al. Central neurocytoma: a synopsis of clinical and histological features. Brain Pathol. 1993;3(3):297-306.

2. Louis DN, Perry A, Reifenberger G, et al. The 2016 World Health Organization Classification of Tumors of the Central Nervous System: a summary. Acta Neuropathol. 2016;131(6):803-820.

3. Nader R, Gragnaniello C, Berta SC, et al, eds. Neurosurgery Tricks of the Trade. New York: Thieme; 2014.

4. Leenstra JL, Rodriguez FJ, Frechette CM, et al. Central neurocytoma: management recommendations based on a 35-year experience. Int J Radiat Oncol Biol Phys. 2007;67(4):1145-1154.

5. Rades D, Fehlauer F, Lamszus K, et al. Well-differentiated neurocytoma: what is the best available treatment? Neuro Oncol. 2005;7(1):77-83.

6. Rades D, Fehlauer F, Schild SE. Treatment of atypical neurocytomas. Cancer. 2004;100(4):814-817.

7. Vasiljevic A, François P, Loundou A, et al. Prognostic factors in central neurocytomas: a multicenter study of 71 cases. Am J Surg Pathol. 2012;36(2):220-227.

8. Rades D, Fehlauer F. Treatment options for central neurocytoma. Neurology. 2002;59(8):1268-1270.

9. Rades D, Schild SE. Treatment recommendations for the various subgroups of neurocytomas. J Neurooncol. 2006;77(3):305-309.

10. Schild SE, Scheithauer BW, Haddock MG, et al. Central neurocytomas. Cancer. 1997;79(4):790-795.

11. Chen KP, Lee CC, Liao CL, et al. Upfront Gamma Knife surgery for giant central neurocytoma. World Neurosurg. 2017;97:751.e15-751.e21.

12. Chen YD, Li WB, Feng J, Qiu XG. Long-term outcomes of adjuvant radiotherapy after surgical resection of central neurocytoma. Radiat Oncol. 2014;9:242.

13. Rades D, Schild SE. Value of postoperative stereotactic radiosurgery and conventional radiotherapy for incompletely resected typical neurocytomas. Cancer. 2006;106(5):1140-1143.

14. Yamanaka K, Iwai Y, Shuto T, et al. Treatment results of Gamma knife radiosurgery for central neurocytoma: report of a Japanese multi-institutional cooperative study. World Neurosurg. 2016;90:300-305.

15. Mozes P, Szanto E, Tiszlavicz L, et al. Clinical course of central neurocytoma with malignant transformation-an indication for craniospinal irradiation. Pathol Oncol Res. 2014;20(2):319-325.

16. Shrivastava A, Mohammed N, Xu Z, et al. Outcomes after gamma knife stereotactic radiosurgery in pediatric patients with Cushing disease or acromegaly: a multi-institutional study. World Neurosurg. 2019;125:e1104-e1113.

17. Yen CP, Sheehan J, Patterson G, Steiner L. Gamma knife surgery for neurocytoma. J Neurosurg. 2007;107(1):7-12.

18. Lin NU, Lee EQ, Aoyama H, et al. Response assessment criteria for brain metastases: proposal from the RANO group. Lancet Oncol. 2015;16(6):e270-e278.
19. Cox JD, Stetz J, Pajak TF. Toxicity criteria of the Radiation Therapy Oncology Group (RTOG) and the European Organization for Research and Treatment of Cancer (EORTC). Int $J$ Radiat Oncol Biol Phys. 1995;31(5):1341-1346.

20. Brandes AA, Amistà P, Gardiman M, et al. Chemotherapy in patients with recurrent and progressive central neurocytoma. Cancer. 2000;88(1):169-174.

21. Kim JW, Kim DG, Kim IK, et al. Central neurocytoma: longterm outcomes of multimodal treatments and management strategies based on 30 years' experience in a single institute. Neurosurgery. 2013;72(3):407-414.

22. Lenzi J, Salvati M, Raco A, et al. Central neurocytoma: a novel appraisal of a polymorphic pathology. Our experience and a review of the literature. Neurosurg Rev. 2006;29(4):286-292.

23. Chen CL, Shen CC, Wang J, et al. Central neurocytoma: a clinical, radiological and pathological study of nine cases. Clin Neurol Neurosurg. 2008;110(2):129-136.

24. Hallock A, Hamilton B, Ang LC, et al. Neurocytomas: long-term experience of a single institution. Neuro Oncol. 2011;13(9):943-949.

25. Park HK, Steven D C. Stereotactic radiosurgery for central neurocytoma: a quantitative systematic review. J Neurooncol. 2012;108(1):115-121.

26. Lee CC, Chou CL, Chen CJ, et al. Stereotactic radiosurgery for hypervascular intracranial tumors. J Neurooncol. 2018;140(3):547-558.

27. Sharma MC, Sarkar C, Karak AK, et al. Intraventricular neurocytoma: a clinicopathological study of 20 cases with review of the literature. J Clin Neurosci. 1999;6(4):319-323.

28. Garcia RM, Ivan ME, Oh T, et al. Intraventricular neurocytomas: a systematic review of stereotactic radiosurgery and fractionated conventional radiotherapy for residual or recurrent tumors. Clin Neurol Neurosurg. 2014;117:55-64.

29. Genc A, Bozkurt SU, Karabagli P, et al. Gamma knife radiosurgery for cranial neurocytomas. J Neurooncol. 2011;105(3):647-657.

30. Karlsson B, Guo WY, Kejia T, et al. Gamma Knife surgery for central neurocytomas. J Neurosurg. 2012;117(suppl):96-101.

31. Pan DH, Lee CC. The management of incidental central neurocytoma. Neurosurg Clin N Am. 2015;26(1):57-66.

32. Chen MC, Pan DH, Chung WY, et al. Gamma knife radiosurgery for central neurocytoma: retrospective analysis of fourteen cases with a median follow-up period of sixty-five months. Stereotact Funct Neurosurg. 2011;89(3):185-193.

33. Martín JM, Katati M, López E, et al. Linear accelerator radiosurgery in treatment of central neurocytomas. Acta Neurochir (Wien). 2003;145(9):749-754.

34. Tanaka H, Sasayama T, Yamashita H, et al. Rapid tumor growth with glial differentiation of central neurocytoma after stereotactic radiosurgery. J Clin Neurosci. 2016;31:188-192.

\section{Disclosures}

Dr. Grills: stock ownership in and board of directors for Greater Michigan Gamma Knife; for non-study-related research funding from Elekta through her institution. Dr. Williams: consultant for Monteris. Dr. Lunsford: consultant for Insightec and DSMB. Direct stock ownership in Elekta.

\section{Author Contributions}

Conception and design: Sheehan, Hung. Acquisition of data: Hung, Lee, Yang, Mohammed, Kearns, Sun, Mathieu, Touchette, Atik, Grills, Squires, Ding, Williams, Yusuf, Woo, Liscak, Hanuska, Shiao, Kondziolka. Analysis and interpretation of data: Hung, Lee, Yang, Mohammed, Xu. Drafting the article: Hung. 
Critically revising the article: Sheehan, Lunsford, Xu. Reviewed submitted version of manuscript: Sheehan, Hung. Approved the final version of the manuscript on behalf of all authors: Sheehan. Statistical analysis: Hung. Administrative/technical/material support: Sheehan. Study supervision: Sheehan.

\section{Supplemental Information}

Online-Only Content

Supplemental material is available with the online version of the article.
Supplement. https://thejns.org/doi/suppl/10.3171/2020.1. JNS191515.

\section{Correspondence}

Jason Sheehan: University of Virginia Health System, Charlottesville, VA. jsheehan@virginia.edu. 